\title{
Resolution of chronic ocular sarcoidosis with antimycobacterial therapy
}

\author{
Bradley W. Richmond ${ }^{1}$, Kyra Richter ${ }^{2}$, Lloyd E. King ${ }^{3}$, Wonder P. Drake ${ }^{2,4}$ \\ 1. Division of Allergy, Pulmonary and Critical Care Medicine, Department of Medicine, Vanderbilt University Medical School, \\ Nashville, United States. 2. Department of Pathology, Microbiology and Immunology, Vanderbilt University School of \\ Medicine, Nashville, United States. 3. Division of Dermatology/Department of Medicine, Vanderbilt University Medical \\ School, Nashville, United States. 4. Division of Infectious Diseases/Department of Medicine, Vanderbilt University Medical \\ School, Nashville, United States.
}

Correspondence: Wonder P. Drake. Address: Department of Medicine, Vanderbilt University School of Medicine, 1161 $21^{\text {st }}$ Avenue, A2200 MCN, Nashville, TN 37232, United States. Email: Wonder.drake@vanderbilt.edu

Received: June 24, 2014

DOI : $10.5430 /$ crim.v1n2p216
Accepted: July 8, $2014 \quad$ Online Published: July 27, 2014

URL: http://dx.doi.org/10.5430/crim.v1n2p216

\section{Abstract}

Ocular and cutaneous sarcoidosis is a chronic manifestation of sarcoidosis that remains difficult to treat. Recent investigations demonstrating efficacy with antimicrobial therapy in pulmonary and cutaneous sarcoidosis have been reported. Here, we report dual clinical improvement in cutaneous and ocular sarcoidosis following administration of oral antimycobacterial therapy.

\section{Keywords}

Sarcoidosis, Antibiotics, Ocular sarcoidosis, T cell proliferation

\section{I ntroduction}

Sarcoidosis is a granulomatous disease of unknown etiology, which affects the lungs in $90 \%$ of affected patients ${ }^{[2]}$. Extrapulmonary sarcoidosis involvement is also common in the United States. Cutaneous and ocular involvement are the most frequent sites, with ocular involvement occurring in approximately one-third of sarcoidosis patients. While anterior involvement can be self-limiting, posterior involvement is more likely to be chronic ${ }^{[4]}$. Most cases of chronic ocular sarcoidosis respond well to immunosuppressive therapy such as methotrexate ${ }^{[3]}$; however, disease control off therapy is difficult to achieve. A growing body of literature supports the therapeutic benefit of tetracyclines on ocular and cutaneous sarcoidosis ${ }^{[1,5,16]}$. A randomized, placebo-controlled, single-blinded trial of the 8 week, oral CLEAR regimen: Levofloxacin $750 \mathrm{mg}$ on day 1, then $500 \mathrm{mg}$ daily, Ethambutol $1200 \mathrm{mg}$ daily, Azithromycin $500 \mathrm{mg}$ on day 1, then 250 mg daily, and Rifampin $300 \mathrm{mg}$ daily, or a comparative placebo regimen was conducted on 30 chronic cutaneous sarcoidosis subjects. In the intention to treat analysis, the CLEAR group has a decrease in lesion diameter of $-8.4 \pm 14.0$ $\mathrm{mm}$, compared to an increase of $+0.07 \pm 3.2$ in the placebo group $(p=0.05)$. A significant reduction in granuloma burden was observed. The CLEAR group also experienced -2.9+2.5 decline in lesion severity, compared to $-0.6+2.1$ among placebo subjects $(p=0.02){ }^{[6]}$. One of the subjects with cutaneous disease had significant, biopsy-proven involvement of the posterior orbit. Herein we report resolution of cutaneous and posterior ocular sarcoidosis for at least one year following completion of an eight week antimycobacterial regimen. 


\section{Case report}

A 61-year old male developed a pruritic, erythematous rash on his forearms bilaterally. The diagnosis of cutaneous sarcoidosis was made after a biopsy of the lesions revealed noncaseating granulomas. The lesions responded only transiently to oral prednisone therapy. Five years after the diagnosis of sarcoidosis, the patient noted increased lacrimation, mild erythema and proptosis of the left eye accompanied by a sensation of ocular fullness with no changes in visual acuity, diplopia, or pain. An ophthalmologist detected a subcutaneous lesion along the left lower lid margin by palpation. A non-contrasted CT scan of the orbits revealed a posterior $2.5 \mathrm{~cm}$ mass surrounding but not infiltrating the left globe (see Figure 1A) with no abnormalities detected in the right orbit and fundus. Biopsy of the ocular mass revealed non-caseating granulomas; stains were negative for fungal, routine and acid-fast bacteria.

A contrasted chest CT scan revealed multiple enlarged hilar and mediastinal lymph nodes, with no lung parenchyma abnormalities (see Figure 1B). The patient denied any pulmonary symptoms and had a normal spirometric evaluation. The patient's lacrimation resolved without treatment so he decided to be monitored while receiving no therapy for sarcoidosis. A CT scan of the orbits and lungs eight months later revealed no interval change in the pulmonary adenopathy or the orbital mass.

The patient elected to participate in a randomized, placebo controlled trial of antimycobacterial therapy for chronic cutaneous sarcoidosis due to severe pruritus. At the time of study entry, his ocular disease was unchanged. The patient was randomized to an eight week, oral regimen consisting of 1) Levofloxacin $750 \mathrm{mg}$ (Day 1) and then $500 \mathrm{mg}$ daily; 2) Ethambutol 1200 mg daily; 3) Azithromycin 500 mg (Day 1) and then 250 mg daily; and 4) Rifampin 300 mg daily. At week four, there was no change in his cutaneous or ocular lesions. After completing eight weeks of the regimen, his pruritis and skin lesions had completely resolved. The patient noted no adverse events, other than his urine color changing to red, which occurred in all patients randomized to drug or placebo. The patient also noted an absence of the sensation of ocular fullness. Palpation along the left lower lid margin revealed normal architecture; he also could not feel the mass along the left lower lid margin. He went to his ophthalmologist, who confirmed the physical examination findings. A non-contrasted orbital CT scan showed complete resolution of his left orbital mass with a normal globe and orbit (see Figure 1C). The patient received no subsequent immunosuppressive or antibiotic therapy. At one year following completion of the antimycobacterial regimen, the patient had no recurrence of his ocular or cutaneous lesions. Review of his $\mathrm{T}$ cell function at baseline compared to after completion of therapy revealed improvement in his proliferative capacity by 25\% (see Figure 2).
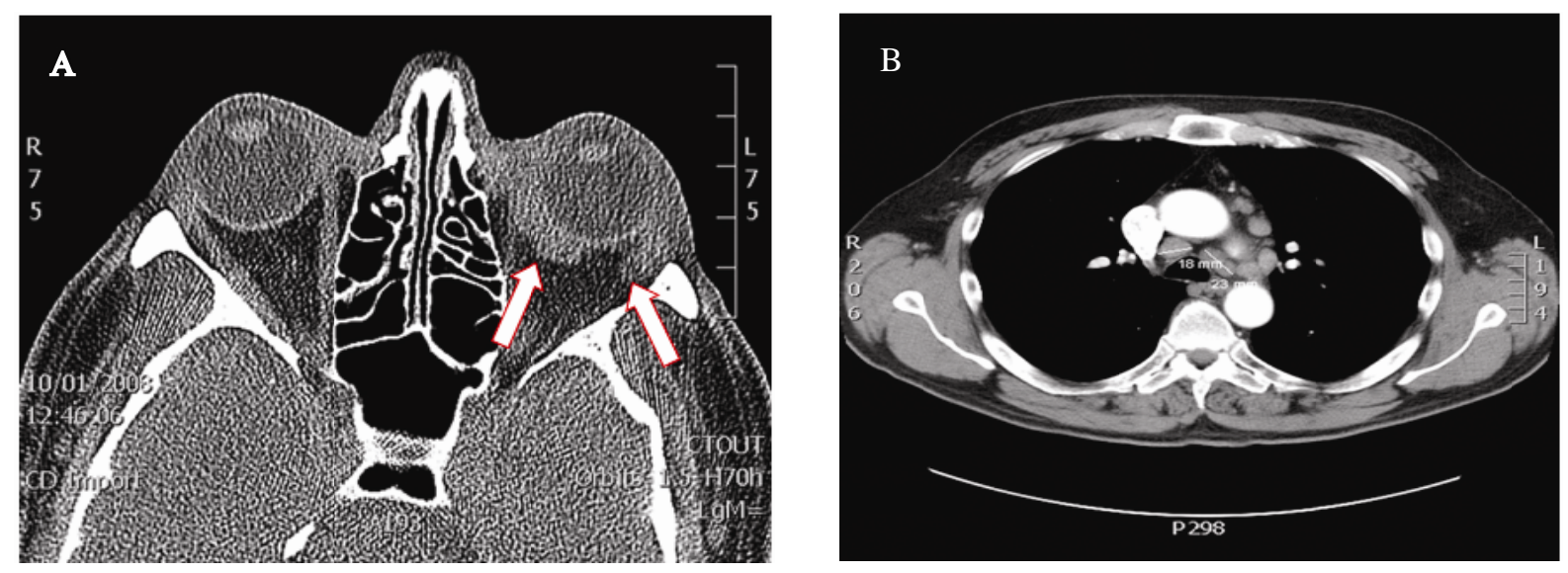

Figure 1A, 1B. Computer tomographic (CT) representation of ocular sarcoidosis and mediastinum at the time of diagnosis and following completion of CLEAR regimen. CT scans of the orbit and lung parenchyma were obtained in a sarcoidosis patient with the sensation of ocular fullness (A, B). Biopsy of the orbital mass confirmed the diagnosis of sarcoidosis. Intervals examinations revealed persistence of the orbital mass. 


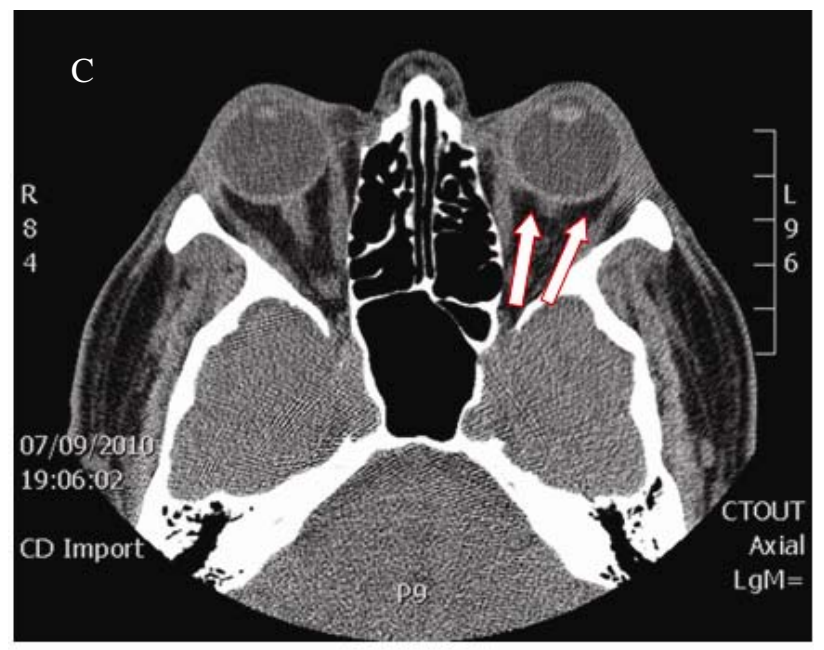

Figure 1C. Computer tomographic (CT) representation of ocular sarcoidosis at the time of diagnosis and following completion of CLEAR regimen. A repeat scan of the orbital mass was obtained following completion of 8 weeks of antimycobacterial therapy. The mass had completely resolved (C).

\section{Baseline}

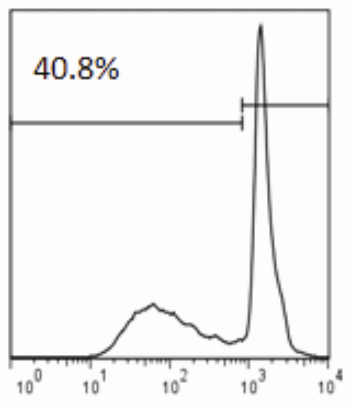

4 weeks

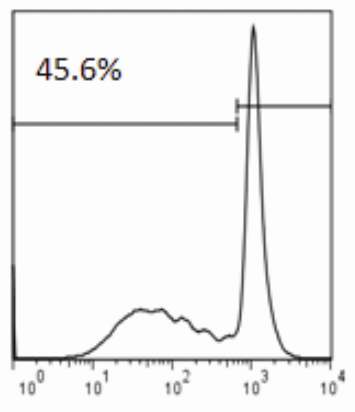

\section{8 weeks}

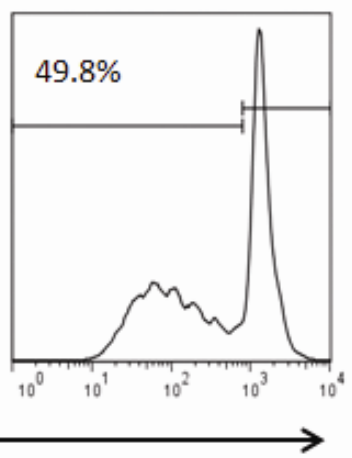

CFSE

Figure 2. CD4+ T cell proliferative capacity. CD4+ T cells were magnetically sorted from PBMC and TCR stimulated in vitro using plate-bound anti-CD3 and soluble anti-CD28 antibodies. The sorted CD4+ T cells were labeled with CFSE, stimulated in vitro 48 hours, cultured an additional 3 days after stimulation, and the percent CD4+ T cell proliferation was determined by flow cytometry. T cell proliferative capacity increased following completion of the CLEAR regimen.

\section{Discussion}

To our knowledge this is the first published case of simultaneous resolution of ocular and cutaneous sarcoidosis following administration of antimycobacterial therapy. It is possible that the patient had a spontaneous remission without treatment since $60 \%-80 \%$ patients with stage I pulmonary disease were reported to have spontaneous pulmonary resolutions ${ }^{[11]}$. Ocular and cutaneous involvement are chronic manifestations of sarcoidosis and much less likely to resolve. The persistence of his ocular and cutaneous disease, of which both were poorly responsive to steroid therapy, makes spontaneous resolution less likely.

A growing body of literature suggests that antimicrobial therapy may benefit chronic sarcoidosis subjects. A retrospective analysis of the effects of minocycline on cutaneous sarcoidosis noted clinical improvement of the lesions ${ }^{[18]}$. The mechanism(s) of action leading to clinical improvement appears to due to immunomodulatory effects. Antimicrobials such 
as azithromycin were shown to be beneficial in non-infectious primary pulmonary inflammatory disorders due to it decreasing neutrophil chemotaxis ${ }^{[19]}$.

An alternative possible mechanism for the observed clinical benefit involves the antimicrobial benefit of the antibiotic regimen. A growing body of literature notes the association of sarcoidosis with microorganisms, such as pathogenic mycobacteria and propionibacteria ${ }^{[8-10,12,13]}$. Pathogenic organisms can induce loss of $\mathrm{T}$ cell function, such as high spontaneous cytokine expression but then through modulation of IL-2 transcription pathway also induce reduced Th1 cytokine expression and proliferative capacity upon stimulation of the T cell receptor (TCR). Sarcoidosis is characterized by $\mathrm{T}$ cell dysfunction; we noted that sarcoidosis subjects with spontaneous clinical remission demonstrate normal $\mathrm{T}$ cell function ${ }^{[15]}$. An improved immune response is assumed to be responsible for the rapid clinical resolution seen after our patient due to the observed improvement in proliferative capacity in this patient after completing the 8 week regimen (see Figure 2). This was similar to the proliferative capacity changes seen in sarcoidosis subjects with spontaneous clinical resolution ${ }^{[15]}$. While independent studies show associations with microorganisms ${ }^{[7,9,10,14,17]}$, additional investigations are necessary in order to delineate if a viable organism is involved in the pathogenesis of cutaneous sarcoidosis. A trial of a larger cohort to confirm these results, define the mechanism(s) of action, determine optimal dosing, as well as patient selection is indicated to define whether the antimycobacterial regimen used will be beneficial for other ocular sarcoidosis patients.

\section{References}

[1] Bachelez H, Senet P, Cadranel J, Kaoukhov A, Dubertret L. The use of tetracyclines for the treatment of sarcoidosis. Arch Dermatol. 2001; 137: 69-73. http://dx.doi.org/10.1001/archderm.137.1.69

[2] Baughman R P, Culver D A, Judson M A. A concise review of pulmonary sarcoidosis. Am J Respir Crit Care Med. 2011; 183: 573-581. http://dx.doi.org/10.1164/rccm.201006-0865CI

[3] Baughman R P, Lower E E, Ingledue R, Kaufman A H. Management of ocular sarcoidosis. Sarcoidosis. Vasc Diffuse Lung Dis. 2012; 29: 26-33.

[4] Baughman R P, Lower E E, Kaufman A H. Ocular sarcoidosis. Semin Respir Crit Care Med. 2010; 31: $452-462$. http://dx.doi.org/10.1055/s-0030-1262213

[5] Doherty C B. Rosen T. Evidence-based therapy for cutaneous sarcoidosis. Drugs. 2008; 68: 1361-1383. http://dx.doi.org/10.2165/00003495-200868100-00003

[6] Drake W P, Oswald-Richter K, Richmond B W, Isom J, Burke V E., Algood H, et al. Oral Antimycobacterial Therapy in Chronic Cutaneous Sarcoidosis: A Randomized, Single-Masked, Placebo-Controlled Study. JAMA Dermatol. 2013. http://dx.doi.org/10.1001/jamadermatol.2013.4646

[7] Dubaniewicz A. Microbial and human heat shock proteins as 'danger signals' in sarcoidosis. Hum Immunol.2013; 74 : 1550-1558. http://dx.doi.org/10.1016/j.humimm.2013.08.275

[8] Dubaniewicz A., Holownia A, Kalinowski L, Wybieralska M, Dobrucki I T, Singh M. Is mycobacterial heat shock protein 16 kDa, a marker of the dormant stage of Mycobacterium tuberculosis, a sarcoid antigen? Hum Immunol. 2013; 74: 45-51. http://dx.doi.org/10.1016/j.humimm.2012.10.007

[9] Ebe Y, Ikushima S, Yamaguchi T, Kohno K, Azuma A, Sato K, et al. Proliferative response of peripheral blood mononuclear cells and levels of antibody to recombinant protein from Propionibacterium acnes DNA expression library in Japanese patients with sarcoidosis. Sarcoidosis.Vasc Diffuse Lung Dis.2000; 17: 256-265.

[10] Ishige I, Usui Y, Takemura T, Eishi Y. Quantitative PCR of mycobacterial and propionibacterial DNA in lymph nodes of Japanese patients with sarcoidosis. Lancet. 1999; 354: 120-123. http://dx.doi.org/10.1016/S0140-6736(98)12310-3

[11] Judson M A. The treatment of pulmonary sarcoidosis. Respir Med. 2012; 106: 1351-1361. http://dx.doi.org/10.1016/j.rmed.2012.01.013

[12] Nunes H, Soler P, Valeyre D. Pulmonary sarcoidosis. Allergy. 2005; 60: 565-582. http://dx.doi.org/10.1111/j.1398-9995.2005.00778.x

[13] Oswald-Richter K. A, Beachboard D C, Seeley, E H, Abraham S, Shepherd B E, Jenkins C A, et al. Dual analysis for mycobacteria and propionibacteria in sarcoidosis BAL. J Clin Immunol. 2012; 32: 1129-1140. http://dx.doi.org/10.1007/s10875-012-9700-5

[14] Oswald-Richter K A, Beachboard D C, Seeley E H, Abraham S, Shepherd B E, Jenkins C A, et al. Dual analysis for mycobacteria and propionibacteria in sarcoidosis BAL. J Clin Immunol. 2012; 32: 1129-1140. http://dx.doi.org/10.1007/s10875-012-9700-5 
[15] Oswald-Richter K A, Richmond B W, Braun N A, Isom J, Abraham S, Taylor, T R, et al. Reversal of Global CD4+ Subset Dysfunction Is Associated with Spontaneous Clinical Resolution of Pulmonary Sarcoidosis. J Immunol. 2013; 190 : 5446-5453. http://dx.doi.org/10.4049/jimmunol.1202891

[16] Park D J, Woog J J, Pulido J S, Cameron J D. Minocycline for the treatment of ocular and ocular adnexal sarcoidosis. Arch Ophthalmol. 2007; 125: 705-709. http://dx.doi.org/10.1001/archopht.125.5.705

[17] Song Z, Marzilli L, Greenlee B M, Chen E S, Silver R F, Askin F B, et al. Mycobacterial catalase-peroxidase is a tissue antigen and target of the adaptive immune response in systemic sarcoidosis. J Exp Med. 2005; 201: 755-767. http://dx.doi.org/10.1084/jem.20040429

[18] Steen T, English J C. Oral minocycline in treatment of cutaneous sarcoidosis. JAMA Dermatol. 2013; 149: $758-760$. http://dx.doi.org/10.1001/jamadermatol.2013.2977

[19] Tamaoki J. The effects of macrolides on inflammatory cells. Chest. 2004; 125: 41S-50S. http://dx.doi.org/10.1378/chest.125.2_suppl.41S 